\title{
Atrophic Rhinitis and Its Surgical Management
}

\author{
${ }^{1}$ Vinit Kumar Sharma, ${ }^{2}$ Rohit Sharma, ${ }^{3}$ JP Purohit, ${ }^{4}$ Sampan Vishth \\ ${ }^{1}$ Assistant Professor, Department of Otolaryngology, SRMS Institute of Medical Sciences, Bareilly, Uttar Pradesh, India \\ ${ }^{2}$ Associate Professor, Department of Otolaryngology, SRMS Institute of Medical Sciences, Bareilly, Uttar Pradesh, India \\ ${ }^{3}$ Professor and Head, Department of Otolaryngology, MLB Medical College, Jhansi, Uttar Pradesh, India \\ ${ }^{4}$ Resident, Department of Otolaryngology, MLB Medical College, Jhansi, Uttar Pradesh, India
}

Correspondence: Vinit Kumar Sharma, Assistant Professor, Department of Otolaryngology, SRMS Institute of Medical Sciences Bareilly, Uttar Pradesh, India, e-mail: drvineetsharma@rediffmail.com

\section{ABSTRACT}

Objective: Clinical evaluation of atrophic rhinitis and comparative study of its surgical treatments.

Materials and methods: This study was carried out in the Department of ORL-HNS, MLB Medical College and Hospital, Jhansi and Department of ORL-HNS, SRMS Institute of Medical Sciences, Bareilly, Uttar Pradesh, India from June 2006 to May 2011 including the cases of atrophic rhinitis. A total of 40 patients of atrophic rhinitis were included in this study. Thirty-two patients had modified Young's nostril closure and eight patients had dermofat graft obliteration of nasal cavity. Preoperative and postoperative assessment of nasal mucociliary flow rate were also assessed by saccharine test. Fifteen cases were kept in control group to compare the nasal mucociliary flow rate results.

Results: The results of both surgical modalities were compared at various intervals for 20 months.

Conclusion: The patients with history of nasal myiasis and septal perforation do better with partial nostril closure while patients, not having history of maggots and septal perforations, had best results with dermofat graft operation. It was seen that after surgical treatment of atrophic rhinitis, either by nostril closure or dermofat grafting, there was improvement in nasal mucociliary flow or, nearly, all patients were symptomatically improved.

Keywords: Nasal myiasis, Atrophic rhinitis, Nasal mucociliary flow rate.

\section{INTRODUCTION}

Atrophic rhinitis is a chronic nasal disease characterized by progressive atrophy of the nasal mucosa and underlying bone of the turbinates and very roomy nasal cavity, the formation of foul smelling thick black or greenish crust, to which attributed the term ozaena. ${ }^{1}$ The earliest complaint is a feeling of dryness in the nose and headache commonly described as an aching behind the eyes. Nasal myiasis occur most commonly in Asia and less commonly in Africa. Nasal myiasis, which is not uncommon in hot and humid climate particularly in India, is also known as Peenash. It is a demoralizing condition of infestation of the nasal cavities by maggots, the larvae of fly (Diptera).

Myiasis can also affect the ear, mouth or larynx and reach its peak in the months of March, April, September, October and November. The majority of sufferers live in bad hygienic conditions and have a source of offensive decaying material, e.g. atrophic rhinitis, chronic sinusitis or chronic suppurative otitis media, which provides a suitable environment for the eggs of the fly to hatch into larvae. ${ }^{2}$ The patient complains of a diffuse swelling around the nose and eyes, nasal obstruction, epistaxis or the presence of maggots coming out of nose. Rhinoscopy reveals a congested edematous mucosa, necrotic material with embedded maggots, ulcerated mucosa or septal perforations. The disease can spread to the paranasal sinuses and via the nasolacrimal duct to lacrimal sac. In the late stages, the nasal bones may be destroyed and death may result from sepsis, meningitis or suicide.

Most of the surgical methods are aimed at reducing the nasal airway. The drying effect of inspired air is a major factor in causation or progression of the disease. Partial obliteration of nasal cavity breaks this viscious cycle, which causes atrophy of the mucosa and widening of the nasal cavity. A review of various surgical treatments is given below:

1. Operation to narrow the nasal cavity

A. Nasal closure operation

i. Anterior nare closure

ii. Choanal occlusion

B. Implantation operation

i. Living tissue implants, like

(a) Autogenous - dermofat, bone, cartilage

(b) Homogenous-placenta

(c) Heterogenous_-bioimplant

ii. Synthetic implants, like acrylic, silicone, teflon, proplast 
2. Denervation operations

A. Sympathectomy
i. Cervical sympathectomy
ii. Stellate ganglion block
iii. Sphenopalatine ganglion block

B. Parasympathectomy-section of greater superficial petrosal nerve.

\section{AIMS AND OBJECTIVES}

1. To study the surgical treatments for atrophic rhinitis and nasal myiasis and compare the follow-up results of modified Young's nostril closure and Girgis dermofat graft implantation.

2. To compare the nasal mucociliary flow rates pre- and postoperatively.

\section{MATERIALS AND METHODS}

This study was carried out in the Department of ORL-HNS, MLB Medical College and Hospital, Jhansi and Department of ORL-HNS, SRMS Institute of Medical Sciences, Bareilly, Uttar Pradesh, India. The work was carried out from June 2006 to May 2011. This study included the cases of atrophic rhinitis and some cases of nasal myiasis. A total of 40 patients were included in this study. Thirty-two patients had modified Young's nostril closure and eight patients had dermofat graft obliteration of nasal cavity. They were reexamined during follow-up at regular intervals and the net outcomes of the above-mentioned modalities were compared. The nasal mucociliary flow rate was also assessed by saccharine test in which, a $0.5 \mathrm{~mm}$ sized particle was placed approximately $1.0 \mathrm{~cm}$ behind the anterior end of inferior turbinate in the sitting position with the head flexed about $10^{\circ}$ to avoid the particle falling back in nasopharynx. The subject was asked to swallow after every 50 seconds and not to sniff or drink. He was asked to tell immediately, as he got the sweet taste of saccharine. The time elapsing until the first experience of sweet taste was recorded as nasal mucociliary flow rate. Fifteen cases were also chosen as control group.

\section{RESULTS}

A. Dermofat graft implantation: The results of dermofat graft implantation show that out of the eight operated cases, one case did not come for follow-up after 6 months. Till 6 months, all the eight cases showed relief. The remaining seven cases maintained good results up to 1 year. After that three cases also lost follow-up in 18 months time, remaining cases maintained good results.

B. Modified Young's operation: The results of 32 cases operated showed that there was complete disappearance of the crusting and foul smelling from the nose. All the cases, during the follow-up periods, showed that patients were relieved from the symptoms.

Patients operated, by either of the above methods, showed significant improvement as assessed by disappearance of visible crusting in nose, pharyngitis sicca and olfactory disturbances. But as a comparative result of the two methods, improvement in symptoms was found better in patients who underwent dermofat graft implantation as found in previous studies also (Table 1 ). ${ }^{3}$

As mucociliary function is also disturbed as a sequelae of atrophic rhinitis, we also assessed the outcome of these two methods by assessing the nasal mucociliary flow rate by saccharin test.

Nasal mucociliary rates as recorded pre- and postoperatively are shown in Table 2. It was seen that there was dramatic improvement in mucociliary flow rate confirming the results of studies in past. ${ }^{4}$ In control group, it was approximately 12.5 minutes. All these patients showed complete improvement in all symptoms.

Table 1: Nasal mucociliary flow rates before and after dermofat grafting

\begin{tabular}{|c|c|c|}
\hline $\begin{array}{l}\text { Total no of } \\
\text { patients }\end{array}$ & Dermofat grafting & $\begin{array}{c}\text { Modified Young's } \\
\text { operation }\end{array}$ \\
\hline 40 & 8 & 32 \\
\hline
\end{tabular}

$\begin{gathered}\text { Table 2: Nasal mucociliary flow rates before and after } \\
\text { modified Young's operation }\end{gathered}$
\begin{tabular}{cc} 
Mean mucociliary flow time (min) \\
\cline { 2 - 3 }
\end{tabular}
\begin{tabular}{lcc} 
Dermofat grafting & $\begin{array}{c}\text { Modified Young's } \\
\text { operation }\end{array}$ \\
\hline Before surgery & 50.4 & 51.2 \\
After surgery & 32.3 & 26.4 \\
\hline
\end{tabular}

Control group-12.5 minutes

\section{DISCUSSION}

In our series, we have done only eight cases of dermofat grafting. This small number was due to choice of the patients between dermofat grafting and nostril closure. We have followed the techniques described by Girgis without any modification, but in two cases, we approached the floor of nasal cavity by incision in nasal vestibule instead of sublabial approach. In the approach via nasal vestibule, bleeding and time for surgery was reduced, because dermofat grafting involves a major operation under general anesthesia with a hospital stay. The immediate postoperative period was quiet stormy in all the eight cases in which sublabial approach was followed, they had swelling of face and pyrexia, While in nasal vestibule approach, swelling of face was minimum. The thigh wound did take time to heal 
up, and in one of our cases, there was wound dehiscence and infection. It required cleaning and dressing for 1 month. Scar on thigh was definitely a marked one, which one feels to avoid as most of the patients are young females. The shrinkage of the graft as stated by Girgis was due to absorption of fat in $25 \%$ and, in some cases, up to $50 \%$ but, in our series, shrinkage was not noticed as a major factor during the follow-up. ${ }^{5}$ All cases, during the 6 months followup period, showed that the floor was raised adequately and only in one case, it was not adequate, probably due to slight shrinkage in graft. Graft rejection was not noticed in any case. Even sloughing and fistula formation as experienced by Girgis $^{6}$ were not seen.

In rest of the 32 cases, Young's operation was done with a slight modification by placing the incision little more anteriorly and converting the technique in double layer closure, i.e. modified Young's operation. The added advantages were safety avoidance of separate wound and the patient's satisfaction of breathing through the nose, and one can perform anterior rhinoscopy with infant-sized ear speculum through the hole. Bilateral operation could also be performed in the same sitting without posing much problems postoperatively and the problems of reopening can be avoided. The operation appears technically a simple one. One does not have to look out for external source or an additional operation for materials for implantation. Most of the cases, it can be performed under local anesthesia with good sedation in adequate doses. Patient experiences mild pain and swelling of the nose for 2 to 3 days. No serious postoperative complications were observed by us. The crusting disappears and the mucosa turns pink very quickly after the closure. Perhaps nostril closure stands as an ideal operation for those unfortunate patients who come with history of maggots, and mutilation of turbinates and nasal tissue by maggots with septal perforation. They do better with partial nostril closure while patients not having history of maggots and septal perforations had best result with dermofat graft operation.

In all of the above-mentioned patients, there was great improvement in nasal mucociliary flow rate after surgery for atrophic rhinitis and all of them were relieved from their symptoms.

Partial nostril closure was done in cases of septal perforation. It is not possible to do another implant operation like dermofat graft in these cases properly. In this series, four patients who came with septal perforation with maggots infestation were subjected to partial nostril closure. All are showing good results inspite of septal perforation. Hence, partial nostril closure stands unique as an ideal operation where septal perforation is present. The partial nostril closure does not pose any problem and is hardly noticeable. In immediate postoperative days, the complaints of dryness of mouth especially after a night's sleep by a few patients were reported. It was due to mouth breathing and is relieved by drinking water. They were also not found more prone to suffer from upper respiratory tract infection. But surely, they are disabled to some extent by the nasal tone of speech.

\section{REFERENCES}

1. Cook Gordon C, Zumla Alimuddin. Manson's Tropical Disease (21st ed), 1600-04, 172732.

2. Warrell David A, Cox Timothy M, Fifth John. Oxford Text Book of Medicine (4th ed), 853-54.

3. Taylor M, Young A. Studies on atrophic rhinitis. J Laryngol Otol 1961;75:574-90.

4. Schonsted-Madsen U, Stoksted P, Christensen PH, KochHenriksen N. Chronic headache related to nasal obstruction. J Laryngol Otol Feb 1986;100(2):165-70.

5. Sinha SN, Sardana DS, Rajvanshi VS. Nine-year review of cases of atrophic rhinitis and its management. J Laryngol Otol 1977;91:591-600.

6. Girgis IH. Surgical management of ozaena by dermofat graft. J Laryngol Otol 1966;80:615-27. 